\title{
Identification of a suitable endogenous control miRNA in bone aging and senescence
}

Authors: Japneet Kaur ${ }^{1,2}$, Dominik Saul ${ }^{1,2}$, Madison L. Doolittle ${ }^{1,2}$, Jennifer L. Rowsey ${ }^{1,2}$, Stephanie J. $\operatorname{Vos}^{1,2}$, Joshua N. Farr ${ }^{1,2}$, Sundeep Khosla ${ }^{1,2}$, and David G. Monroe ${ }^{1,2^{*}}$

Affiliations:

${ }^{1}$ Department of Medicine, Division of Endocrinology, Mayo Clinic College of Medicine, Rochester, MN 55905, USA.

${ }^{2}$ Robert and Arlene Kogod Center on Aging, Rochester, MN 55905, USA.

*To whom correspondence should be addressed:

David G. Monroe, PhD, Department of Medicine, Division of Endocrinology, Mayo Clinic College of Medicine, Guggenheim 7-11A, 200 First Street SW, Rochester, MN 55905, USA. Telephone: 507-5386517; Fax: 507-293-3853; E-mail: monroe.david@mayo.edu.

Disclosure: The authors have nothing to disclose. 


\section{ABSTRACT}

Objective

MicroRNAs (miRNAs) are promising tools as biomarkers and therapeutic agents in various chronic diseases such as osteoporosis, cancers, type I and II diabetes, and cardiovascular diseases.

Considering the rising interest in the regulatory role of miRNAs in bone metabolism, aging, and cellular senescence, accurate normalization of qPCR-based miRNA expression data using an optimal endogenous control becomes crucial.

Methods

We used a systematic approach to select candidate endogenous control miRNAs that exhibit high stability with aging from our miRNA sequence data and literature search. Validation of miRNA expression was performed using qPCR and their comprehensive stability was assessed using the RefFinder tool which is based on four statistical algorithms: GeNorm, NormFinder, BestKeeper, and comparative delta CT. The selected endogenous control was then validated for its stability in mice and human bone tissues, and in bone marrow stromal cells (BMSCs) following induction of senescence and senolytic treatment. Finally, the utility of selected endogenous control versus U6 was tested by using each as a normalizer to measure the expression of miR-34a, a miRNA known to increase with age and senescence.

\section{Results}

Our results show that Let-7f did not change across the groups with aging, senescence or senolytic treatment, and was the most stable miRNA, whereas U6 was the least stable. Moreover, using Let-7f as a normalizer resulted in significantly increased expression of $m i R$-34a with aging and senescence and decreased expression following senolytic treatment. However, the expression pattern for miR-34a reversed for each of these conditions when U6 was used as a normalizer.

\section{Conclusions}

We show that optimal endogenous control miRNAs, such as Let-7f, are essential for accurate normalization of miRNA expression data to increase the reliability of results and prevent misinterpretation. Moreover, we present a systematic strategy that is transferrable and can easily be used to identify endogenous control miRNAs in other biological systems and conditions.

Keywords: microRNA; Let-7f; miR-34a; osteoporosis; senolytics 


\section{INTRODUCTION}

MicroRNAs (miRNAs) are a class of evolutionarily conserved short non-coding RNAs ( 22 nucleotides (nt)) that bind to the 3'-untranslated regions (3'-UTR) of target mRNAs, resulting in mRNA degradation and suppression of translation (Bartel, 2004; Bushati \& Cohen, 2007). Following the discovery of lin-4, the first miRNA to be discovered that repressed lin-14 message in C. elegans, thousands of miRNAs have been identified in various plants and animal species including humans that regulate a wide range of biological functions such as cell proliferation, apoptosis, regeneration, and metabolism (Bartel, 2004; Lee et al., 1993; Wightman et al., 1993). Moreover, their ability to circulate throughout the body in cell-free encapsulated vesicles called extracellular vesicles or exosomes (30-150nm-diameter) have made them potent signaling molecules, disease biomarkers, and essential prognostic and therapeutic tools in chronic age-related diseases such as osteoporosis, cardiovascular diseases, type I and II diabetes, and cancers (Bottani et al., 2019; Condrat et al., 2020; Foessl et al., 2019; Januszewski et al., 2021; Mandourah et al., 2018; Margaritis et al., 2021; Materozzi et al., 2018).

Aging is a major risk factor for most chronic diseases and is characterized by progressive multi-organ deterioration and tissue dysfunction, and long-term accumulation of permanently growth-arrested 'senescent cells' in various tissues (Franceschi et al., 2018; Jaul \& Barron, 2017; Kaur et al., 2019; Kaur \& Farr, 2020; Khosla et al., 2020; McHugh \& Gil, 2018). Originally described by Hayflick in 1961, cellular senescence is a cell fate that results in irreversible cell cycle arrest in response to a stressor, such as DNA damage, oncogenic insult, and reactive oxygen species, etc., along with activation of tumor

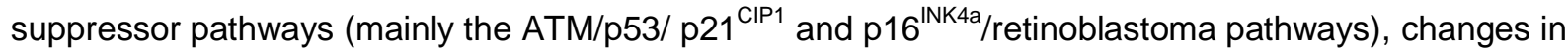
chromatin organization, resistance to apoptosis, and excessive production of pro-inflammatory cytokines (the senescence-associated secretory phenotype (SASP)) that induce senescence in neighboring healthy cells. Senescent cells are characterized by their flattened morphology and increased senescenceassociated $\beta$-galactosidase (SA- $\beta$-Gal) activity and mRNA expression of $p 16^{\text {ink4a }}$ and $p 21^{\text {cip } 1}$ tumor suppressor genes (Baker et al., 2011; Khosla et al., 2020; Kirkland \& Tchkonia, 2017). Age-related accumulation of senescent cells is one of the main causative factors of osteoporosis that can be impeded by selectively targeting these cells using senolytic drugs such as a combination of dasatinib plus quercetin $(D+Q)$ that specifically eliminates senescent cells via senolysis (seno = senescence; lysis = kill) to thereby extend healthspan, and result in decreased number of SA- $\beta-G a l$ positive cells and reduction in $p 16^{\operatorname{lnk} 4 a}$ and $p 21^{C i p 1}$ gene expression (Farr et al., 2017; Zhu et al., 2015). Therefore, there is mounting interest in developing senolytics for alleviating age-related tissue dysfunction and to identify appropriate techniques to monitor senolysis in various biological settings.

Recently, miRNAs have emerged as key regulators of bone remodeling, aging, and cellular senescence, and are known to be mechanistically involved in regulating these conditions (Grillari et al., 2021; Potter et al., 2021; Thalyana \& Slack, 2012). Despite the rapid increase in the availability of high-throughput deep sequencing for characterization of miRNA transcriptome, qPCR remains the gold-standard and most 
widely used method for measuring miRNA expression (Donati et al., 2019; Drobna et al., 2018). However, the accuracy of their expression data obtained via qPCR is highly dependent on the choice of an appropriate endogenous normalizer or control. MicroRNA endogenous controls need to be assessed for each experiment separately since their expression varies depending on their tissue of origin and the disease state or condition being tested (Landgraf et al., 2007). Generally, a suitable endogenous control is a gene or a combination of genes that is structurally similar to the genes being tested, is highly stable, and is relatively abundantly expressed across the different models and conditions being tested (Drobna et al., 2018). Most of the studies investigating miRNAs use small nuclear RNAs (snRNAs) such as U6 as their endogenous normalizer (Beyer et al., 2015; He et al., 2013; H. Li et al., 2018; T. Xu et al., 2020). However, the structural differences between snRNAs (150nt) and miRNAs (20-24nt) can impact their isolation yield, reverse transcription, and amplification efficiencies, leading to inaccurate and unreliable results and possible misinterpretation of the mechanistic and therapeutic roles of the miRNAs being tested (Drobna et al., 2018).

With rising interest in the role of miRNAs in bone aging and cellular senescence, a pertinent question is which miRNA will serve as an optimal endogenous normalizer. To answer this question, we used a stepwise approach to identify a suitable endogenous control miRNA from miRNA sequence data and existing literature that remains stable with aging in bone tissue of mice and humans and with senescence as well as in response to senolytic treatment in culture. Following this, the applicability of the selected miRNA control versus $U 6$ was tested by using each as a normalizer to measure the expression of miR$34 a-5 p$, a miRNA well known in the literature to increase with aging and senescence in various tissues and cells and predicted to target $p 16^{\text {ink } 4 a}$ and $p 21^{\text {cip } 1}$ in humans as per our bioinformatic analyses (Fulzele et al., 2019; Q. Li et al., 2019; Zhang et al., 2018). The expression of miR-34a was evaluated in bone biopsies from young and old women, previously shown to have increased expression of the senescencerelated genes $p 16^{\text {ink4a }}, p 21^{\text {cip } 1}$, and a subset of SASP factors, and in mouse BMSCs following induction of senescence and senolytic treatment (Farr et al., 2016). This study was driven by our observation on the lack of stability of $U 6$ across different biological conditions and its widespread use as an endogenous normalizer in the absence of an optimal endogenous control miRNA.

\section{Materials and Methods}

Bone tissue samples (mouse and human). All mouse studies were conducted in accordance with $\mathrm{NIH}$ guidelines and approved by the Institutional Animal Care and Use Committee at the Mayo Clinic. The vertebrae isolated from both 6-month-old and 24- month-old male C57BL/6N mice ( $n=10 /$ group) were cleaned of associated muscle and connective tissues, minced into $\sim 1$-mm pieces and sequentially digested twice for 30-min in endotoxin-free collagenase (Liberase; Roche Diagnostics GmbH, Mannheim, Germany) to obtain osteocyte-enriched bone samples. These were then homogenized in QIAzol reagent (Qiagen, Valencia, CA) for total RNA isolation and used for miRNA sequencing. (Farr et al., 2016). Similarly, vertebrae from young (6-month) and old (24-month) male and female mice prepared in the 
same way were used for RT-qPCR analyses. The metaphysis and diaphysis were also cleaned of the muscle and connective tissue and homogenized in QIAzol reagent (Qiagen). The human bone biopsy samples used in this study were as described in previous publications (Farr et al., 2015, 2016; Fujita et al., 2014); they were small needle bone biopsies isolated from the posterior iliac crest procured from 10 young premenopausal women (mean age \pm SD, $27 \pm 3$ years; range 23 to 30 years) and 10 old postmenopausal women (78 \pm 5 years; range 72 to 87 years). Postmenopausal status was established by the absence of menses for $>1$ year and serum follicle stimulate hormone levels $>20 \mathrm{IU} / \mathrm{L}$. Extensive exclusion criteria for these patients and the study protocol are as previously described (Farr et al., 2015). The RNA from these bone biopsy samples was reanalyzed for miRNA expression. All human studies were approved by the Mayo Clinic Institutional Review Board.

Cell culture. Bone marrow stromal cells (BMSCs) were isolated from 3-month-old and 33-month-old mice C57BL/6N mice after removing the epiphyseal growth plates from the tibias and femurs and flushed with Dulbecco's Modified Eagle Medium (DMEM, ThermoFisher Scientific, Waltham, MA, USA) supplemented with $1 \times$ antibiotic/antimycotic (ThermoFisher Scientific), $1 \times$ Glutamax, and 15\% (v/v) fetal bovine serum (GE Healthcare Life Sciences HyClone Laboratories, Logan, UT). Half media change was done on day 3 and the cells were plated for the experiment on day 7 at a cell density of $10^{4}$ cells $/ \mathrm{cm}^{2}$ in 12 -well tissue culture plates and allowed to grow to $70-80 \%$ confluence. To induce senescence, the cells from the 3month-old mice were then treated with $20 \mathrm{uM}$ of etoposide (MilliporeSigma, St. Lous, MO) or vehicle $(0.1$ \% DMSO) for 48 hours followed by maintenance in growth media for 6 days. For senolytic treatment, cells from 33-month-old mice were treated with $0.2 \square \mu \mathrm{M}$ dasatinib (LC Laboratories, Woburn, MA) and 20 $\square \mathrm{MM}$ quercetin (Sigma-Aldrich, St. Louis, MO) or vehicle (DMSO) for $24 \square \mathrm{h}$, washed, and allowed to recover for 1 additional day (Zhou et al., 2021). Cells were lysed in QIAzol reagent (Qiagen) for RNA isolation or fixed with $4 \%$ paraformaldehyde (PFA) for SA- $\beta$-Gal assay.

SA- $\beta$-Gal Assay. Cellular SA- $\beta$-Gal activity was assayed as previously described (M. Xu et al., 2015). In brief, following fixation, the BMSCs were washed three times with 1x PBS before being incubated in SA$\beta$-Gal activity solution ( $\mathrm{pH} \mathrm{6.0)}$ ) at $37^{\circ} \mathrm{C}$ for $16-18$ hours. The enzymatic reaction was stopped by washing cells or tissues three to five times with ice-cold $1 \times$ PBS.

miRNA-sequencing. miRNA sequence analysis was performed on the osteocyte-enriched bone samples obtained from collagenase digested vertebrae of young ( $n=10,6$-month) and old ( $n=10,24-$ month) C57BL/6N male mice using previously described methods (Farr et al., 2016). MicroRNAs with raw read counts less than 4 in both the groups were classified as unreliable and deleted. NormFinder analyses was done using $R$ (4.0.1) to assess the stability scores of miRNA expression across the groups and then the miRNAs were sorted to select the ones with the highest normalized counts. The predicted target 
mRNAs for miR-34a-5p in humans were determined using miRNet (https://www.mirnet.ca/miRNet/home.xhtml).

Analyses of RT-qPCR expression stability. For assessing the expression stability of the RT-qPCR data, the median CT values (from triplicates) collected for each candidate endogenous control miRNA for each sample were averaged and used as the input data in RefFinder tool (https://www.heartcure.com.au/reffinder/) which generated a comprehensive stability ranking and individual ranking for each of the four algorithms: GeNorm (Vandesompele et al., 2002), NormFinder (Andersen et al., 2004), BestKeeper (Pfaffl et al., 2004), and comparative delta CT (Silver et al., 2006).

qPCR analyses of mRNA and miRNA assays. Total RNA (125ng) was used to generate cDNA using the High-Capacity cDNA Reverse Transcription Kit (Applied Biosystems, Carlsbad, CA) according to the manufacturer's instructions. qPCR analysis was performed using the ABI Prism 7900HT Real-Time System instrument (Applied Biosystems) with SYBR Green reagent (Qiagen) for mRNA and as previously described (Farr et al., 2015). Data normalization was performed based on 5 reference genes (Actb, Gapdh, Polr2a, Rp/13a, Tuba1a) depending on their stability and threshold calculations are as previously described (I. Mödder et al., 2011). The oligonucleotide sequences for the genes measured in this study were designed using the Primer Express program (Applied Biosystems) and are available upon request. For the miRNA expression data, total RNA (30ng) including the miRNA fraction was reverse transcribed using the miRCURY LNA RT Kit (Qiagen) as per manufacturer's protocol. The individual miRNA assays used in this study were purchased from Qiagen and used with the miRCURY LNA miRNA PCR Starter Kit (Qiagen) according to the manufacturer's instructions. Data normalization was performed using the Let-7f and RNU6B (U6) small nucleolar RNA (snRNA) as specified in Results.

Data analysis. miRNA expression levels of the candidate endogenous controls were determined by CT values measured in triplicates. The fold-changes were determined using the relative quantification method $\left(2^{-\Delta \Delta C t}\right)$ with the selected endogenous control. All generated quantitative data was presented as the mean \pm SE (unless otherwise specified) and differences were analyzed using t-test analysis with $p<0.05$ (two-tailed) considered to be statistically significant. Statistical analyses were performed using the Statistical Package for the Social Sciences for Windows, Version 25.0 (SPSS, Chicago, IL).

\section{Results}

\section{Identifying suitable endogenous controls from the miRNA sequence dataset}

We utilized a systematic strategy to identify suitable endogenous controls from our miRNA sequence dataset obtained from the osteocyte-enriched bone samples of young (6-month) and old (24-month) mice ( $n=10$ group) (Fig. 1). To select the optimal number of miRNAs from the total of 633 expressed in our 
dataset, we extended the approach followed by Drobna et al. and used their 'iterative analysis of stability' algorithm based on NormFinder to shortlist the best 100 miRNAs with the highest stability from our dataset (Drobna et al., 2018). A standard NormFinder analysis identified the most stable miRNAs and then the signals of these miRNAs were averaged with the remaining $\mathrm{N}-1$ miRNAs individually generating a stability value. Generally, the addition of a miRNA is expected to decrease the stability score, thereby indicating higher stability.

We established our average NormFinder stability scores for each iteration and the minimum score representing the best set of miRNAs for a particular size (Fig. 2a). For our dataset, even a single miRNA had a low minimal stability score $(<0.1)$ and the addition of a second miRNA barely lowered this score further, although the addition of a third miRNA lowered the score to 0.00 . Thus, based on these results we speculated that we need anywhere between 1-3 control miRNAs to normalize our dataset; however, we conservatively selected 3 miRNAs as candidate endogenous controls for initial validation. Due to the relatively low read counts of many of these miRNAs, we focused our selection on miRNAs with high normalized counts and relatively low stability scores. Using these criteria, we selected Let-7i-5p (avg. normalized counts=87679.671; stability score $=0.07$ ), Let-7f-5p (avg. normalized counts $=47232.569$; stability score $=0.16$ ) and miR-185-5p (avg. normalized counts $=971.411$; stability score $=0.09$ ) as our candidate endogenous control miRNAs.

To widen the scope of our study, we performed an extensive literature search for additional miRNAs that were reported to be stable in different biological systems and conditions and incorporated those into our analyses. Our final set of candidate endogenous control miRNAs consisted of: Let-7i, Let-7f and miR185-5p selected from miRNA sequence dataset and miR-25-3p (Chen et al., 2016; Drobna et al., 2018; Niu et al., 2016), -320a (Allen-Rhoades et al., 2015; Schlosser et al., 2015), -26a-5p (Y. Li et al., 2015), 16-5p (Drobna et al., 2018; Song et al., 2012; Wang et al., 2015), and Let-7a-5p (Drobna et al., 2018) from the published literature, and each of these were compared to U6 for their stability (Table 1).

\section{Evaluating the stability of selected endogenous control miRNAs with qPCR}

We next tested the stability of these candidate endogenous control miRNAs in the osteocyte-enriched samples of young (6-month) and old (24-month) male and female mice ( $n=6$ per group (equal number of males and females)). As shown in Figure $2 b$, the average of the raw CT values of all miRNAs remains unchanged with aging ( $p>0.05$ ) except for miR-16-5p which increased significantly with aging, and therefore was eliminated from further analyses.

\section{Selection of Let-7f as the endogenous control miRNA in aging}

To select a stable endogenous control, we used four algorithms: GeNorm, NormFinder, BestKeeper, and comparative Delta CT, available publicly at https://www.heartcure.com.au/reffinder/ which ranked our candidate endogenous controls as per their stability. Finally, the RefFinder tool was used to generate a 
cumulative stability ranking for these miRNAs based on their average stability calculated by each of these four algorithms, with a lower ranking indicating a higher stability.

GeNorm: This algorithm ranked the selected miRNAs in order of their decreasing stability (M-value) with 1.5 considered as the upper limit for a stable miRNA. Although in our analyses all the miRNAs had an Mvalue of less than 1.5, miR-185-5p exhibited the highest stability whereas U6 was the least stable (Fig. 3a).

NormFinder: This algorithm uses variation between and within the groups to assess the stability of the candidate miRNAs. It ranked miR-320a as the most stable and U6 as the least stable endogenous control (Fig. 3b).

BestKeeper: As per this algorithm, Let-7f was the most stable control miRNA. Moreover, the SD value of U6 $(S D=3.06)$ surpassed the $S D$ threshold $(S D=1.0)$ utilized by this algorithm and was ranked as least stable (Fig. 3c).

Comparative Delta CT: Consistent with the results of BestKeeper, this algorithm ranked Let-7f as the most stable and $U 6$ as the least stable gene (Fig. 3d).

RefFinder: This algorithm created a final comprehensive stability ranking for the selected miRNAs based on the divergent rankings produced by each of the four algorithms using their geometric mean values. Based on this analysis, Let-7f was the most stable and U6 remained the least stable endogenous control (Fig. 3e).

Based on these results and those of iterative analyses indicating the sufficiency of using a single miRNA as an endogenous control for our dataset, we selected Let-7f-5p as our endogenous control miRNA and validated its stability with aging and senescence in bone tissue and cells.

\section{Validation of Let-7f as a suitable endogenous control in aging bone tissue of mice and humans}

We next tested the stability of Let-7f at the metaphyses and diaphyses of the femur in young (6-month) and aged (24-month) male and female mice ( $n=3 / g r o u p / s e x)$. Consistent with the results at the vertebrae (trabecular bone site), Let-7f was stable with aging across both predominantly cortical bone sites.

Moreover, Let-7f was also stable in needle bone biopsies (containing both trabecular and cortical bone as well as bone marrow elements) from the posterior iliac crest of young (mean $\pm S D=27 \pm 3$ years) and old (mean $\pm S D=78 \pm 6$ years) healthy women ( $n=10$ /group) who had significantly higher expression of senescence-related genes with aging (Fig. 3f).

Validation of Let-7f as a suitable endogenous control in BMSCs following induction of senescence and senolytic treatment 
The stability of Let-7f was also tested in non-senescent (vehicle-treated) and senescent (etoposidetreated) BMSCs as well as in senescent BMSCs treated with the senolytic cocktail - dasatinib (D; an FDAapproved tyrosine kinase inhibitor) plus quercetin (Q; a flavanol present in many fruits and vegetables). Senescence was induced in BMSCs using etoposide, a chemotherapeutic agent capable of causing DNA damage, and was confirmed with an increased mRNA expression of $p 16^{\text {ink4a }}(28.5$-fold; $p<0.001)$ and $p 21$ (8.3-fold; $p<0.001$ ) and increased SA- $\beta$-Gal activity compared to the vehicle-treated cells (Fig. $4 a, b)$. Moreover, treatment of senescent BMSCs with $D+Q$ was followed by downregulation of $p 16^{\text {ink4a }}(0.2$-fold; $p<0.001)$ and $p 21$ (0.5-fold; $p<0.001)$ gene expression and fewer $S A-\beta$-Gal positive cells compared to the vehicle-treated cells, thus confirming the senolytic effects of $D+Q$ (Fig. $4 d$, e). Let-7f remained stable both with senescence and $D+Q$ treatment in BMSCs (Fig. 4c, f).

\section{Testing the translational application of Let-7f versus $U 6$ for data normalization in humans}

To test the application scope of Let-7f as a control miRNA versus U6, we measured the expression of miR-34a-5p in human bone biopsy samples from young vs old women and in BMSCs following induction of senescence and after administration of senolytics ( $D+Q)$. When miR-34a was measured using Let-7f as the endogenous normalizer, its expression increased with aging in human bone biopsies (Fig. 5a; 1.6 fold; $p=0.001$ ) and with senescence in BMSCs (Fig. $5 b ; 1.5$-fold; $p=0.032$ ) and decreased following treatment with $D+Q$ (Fig. 5c; 0.5-fold; $p=0.03$ ). However, when U6 was used as the endogenous control, miR-34a did not change significantly with aging (Fig. 5a; 0.1 -fold; $p=0.323$ ) and senescence (Fig. 5b; 0.9fold; $p=0.91$ ) and showed an upregulated trend following treatment with $D+Q$ (Fig. 5c; 6.2-fold; $p=0.08$ ).

\section{Discussion}

Aging is characterized by a gradual physiological decline resulting in impairment of physical function and increased risk of mortality. Cellular senescence is one of the 'hallmarks of aging' that significantly contributes to the aging process and its related phenotype (López-Otín et al., 2013). Multiple studies have shown that senescent cells accumulate with aging in various tissues, including bone and various marrow cell populations, subsequently leading to osteoporosis and its related fractures (Farr et al., 2016, 2017). MicroRNAs have been identified as critical regulators of bone remodeling, aging, and cellular senescence, that mostly downregulate gene expression by binding to the 3'-UTR of the target mRNAs (Bushati \& Cohen, 2007; Pignolo et al., 2021). Considering the rising interest in miRNAs as biomarkers, diagnostic and therapeutic agents, and their mechanistic role in the regulation of various biological processes and diseases, inter-experimental reproducibility and comparability of their qPCR-based expression data across various studies is crucial. Thus, the selection of an appropriate endogenous control becomes important for data normalization to generate reliable, comparable, and reproducible results.

In this study, we selected a set of candidate endogenous control miRNAs from our aging-related miRNA sequence dataset and literature search and compared them to U6, a widely used control gene in miRNA- 
related studies. These selected candidate control miRNAs were then validated and ranked for their stability based on four algorithms, GeNorm, NormFinder, BestKeeper, and Comparative Delta CT, and finally, the RefFinder tool created a final stability ranking using their geometric mean values. Although each algorithm established a different miRNA as 'most stable' (GeNorm: miR-185; NormFinder: miR320a; BestKeeper and Delta CT: Let-7f ), U6 remained the least stable control gene across all algorithms. Our results depicting the instability of $U 6$ are consistent with previous studies where $U 6$ is regarded as a highly unstable gene in various conditions (Lou et al., 2015; Xiang et al., 2014). Based on our analyses, we selected Let-7f as our endogenous control miR for further validation.

Let-7f remained stable at the metaphysis and diaphysis of the femur in mice and in human bone biopsy samples from young versus old women with significantly higher mRNA expression of $p 16^{\text {ink } 4 a}$ and $p 21$, as well as a subset of SASP markers. These results indicated the extended applicability of Let-7f as a control miRNA in senescence-related conditions. We further reported that Let-7f remained stable in BMSCs following induction of senescence with etoposide and in senescent BMSC after treatment with a senolytic cocktail $(D+Q)$, thus expanding the scope of utility of Let-7f as a control miRNA to both bone aging, senescence, and monitoring of the selective elimination of senescent cells - i.e., "senolysis". We then tested the utility of Let-7f as a normalizer in comparison to U6 by using each to normalize the expression of miR-34a, a miRNA well established in the literature to increase with aging and senescence in various cells and tissues. The expression of miR-34a was measured in young versus old human bone biopsy samples, and in BMSCs following induction of senescence and treatment with senolytics.

Interestingly, normalization using Let-7f resulted in an expected and significant increase in miR-34a expression with aging and senescence and a decrease in its expression after treatment with $D+Q$. However, following normalization to $U 6$, the expression pattern of miR-34a changed where it decreased with aging and senescence and increased in response to senolytic treatment. Although the fold-changes in each of these conditions were quite large, particularly with aging and senolytic treatment, the results showed no statistical significance owing to the large intra- and inter-group variability. These results are reflective of the highly unstable nature of $U 6$ and the corresponding misinterpretation of data that can occur on using an unsuitable endogenous normalizer.

Most studies evaluating miRNAs in bone use U6 or another snRNA as a control, whereas few studies have investigated condition-specific endogenous control miRNAs in bone tissue or cells. Chen et al. reported miR-25-3p as the most stable miRNA in serum samples from osteoporotic rats, monkeys and women, and with osteoblast and osteoclast differentiation (Chen et al., 2016). Additionally, miRs-103a-3p and $-22-5 p$ have been reported to be highly stable in extracellular vesicles released from cartilage cells, subcutaneous adipose tissue and BMSCs of osteoarthritic patients and miR-191-5p is shown to have high stability in human bone marrow stromal cell lines and primary cells (Costé \& Rouleux-Bonnin, 2020; Ragni et al., 2021). The results of each of these studies are unique and different from our results due to differences in methodologies, and the disease states and conditions being tested. Although the selection 
of our candidate control miRNAs was partly based on miRNA sequence data, we validated our results using qPCR, which is essential as the operating principles used for quantification by the two methods are different. MicroRNA sequencing is based on raw read counts of each transcript whereas qPCR is based on hybridization and amplification of primers and probes. Moreover, qPCR remains the most widely accepted and used method for quantifying gene expression to date (Livak \& Schmittgen, 2001; Rajeevan et al., 2001). Furthermore, our results using Let-7f or U6 to measure the change in expression of miR34a under various conditions re-emphasized our notion that an unstable endogenous control can lead to faulty findings and misinterpretation of results thereby hindering the reproducibility of the study and its comparison across the literature.

A limitation of this study is that although we validated Let-7f in BMSCs following induction of senescence and senolytic treatment, our miRNA sequence data was derived from young and aged mice. It has been shown previously that senescent cells accumulate with aging; however, we acknowledge that our aged bone tissue samples were not a pure senescent cell population. For our study design, the ideal selection of candidate control miRNAs should be made from three different miRNA sequence datasets - young versus aged, non-senescent vs senescent BMSCs, and vehicle versus senolytic treated BMSCs. However, conducting the experiments at such a vast scale was beyond the scope of the current study, which opens the possibility that another miRNA by itself or in combination with Let-7f may be as or even more stable than Let-7f alone. Another limitation is that we were not able to compare our endogenous control miRNA other small RNAs such as SNORD44, SNORD48, SNORD68, SNORD116 etc., that have been used as control genes for miRNA related studies (Bignotti et al., 2016; Masè et al., 2017). Although like U6, these small RNAs are structurally different from miRNAs which is a caveat for their use as an endogenous control. Therefore, future studies will be needed to assess the stability of these and other small RNAs.

In conclusion, we used a systematic approach to identify Let-7f as a suitable endogenous control miRNA that remains stable in the bone tissue of mice and humans with aging and in BMSCs with senescence and in response to senolytic treatment and confirmed its translational applicability by using it as a normalizer to assess the expression of a well-established aging and senescence-associated miRNA. 


\section{HIGHLIGHTS}

- Let-7f is the most stable miRNA with aging at cortical and trabecular bone sites in young and old mice, and in human bone tissue samples from young and old women with high levels of senescence and SASP related genes.

- Let-7f maintains its stability in BMSCs following induction of senescence and in senescent BMSCs after treatment with senolytic cocktail of dasatinib plus quercetin.

- When Let-7f is used as a normalizer versus U6, miR-34a expression increases with aging in human bone tissue samples and in BMSCs from mice following induction of senescence and decreases in senescent BMSCs after treatment with dasatinib plus quercetin. 


\section{FIGURE LEGENDS (use color for all figures)}

Figure 1. Schematic diagram of the study design (designed using Biorender.com).

Figure 2. Selection of candidate endogenous control miRNAs. (A) NormFinder mean and minimal stability scores relative to the number of miRNAs iterated. The plot has been limited to first 50 iterations for visualization, beyond which no definite changes were observed. (B) RT-qPCR analysis of the candidate endogenous control miRs and U6 in the vertebrae of young (6-month) and old (24-month) male and female mice ( $n=6 /$ group). Data represent mean \pm SEM. ${ }^{*} p<0.05 ;{ }^{* *} p<0.01 ;{ }^{* \star *} p<0.001$ (independent samples t-test).

Figure 3. Let-7f is the most stable miRNA with aging in bone tissue of mice and humans. Stability ranking of candidate endogenous control miRNAs by (A) GeNorm, (B) NormFinder, (C) BestKeeper, (D) Comparative Delta CT, and (E) RefFinder. (F) RT-qPCR analysis of the Let-7f in femur metaphysis and diaphysis of young (6-month) and old (24-month) male and female mice ( $n=6 /$ group) and in small needle bone biopsies obtained from the posterior iliac crest of 10 young (mean \pm SD; $27 \pm 3$ years) and 10 old (mean \pm SD; $78 \pm 6$ years) healthy female volunteers. Data represent mean \pm SEM. ${ }^{*} p<0.05 ;{ }^{* *} p<0.01$; ${ }^{\star * *} \mathrm{p}<0.001$ (independent samples t-test).

Figure 4. Let-7f remains stable in BMSCs following induction of senescence and with senolytic treatment. (A) Representative images of the SA- $\beta$-Gal stained BMSCs treated with vehicle (DMSO) and Etoposide (20uM) to induce senescence (magnification, 10X; $n=3 /$ group). RT-qPCR analysis of (B) $p 16^{\text {ink } 4 a}$ and $p 21^{\text {Cip } 1}$, and (C) Let-7f-5p, in BMSCs treated with vehicle (DMSO) and Etoposide (20uM); gene expression was denoted as fold-change relative to vehicle ( $n=3 /$ group). (D) Representative images of the SA- $\beta-$ Gal stained BMSCs treated with vehicle (DMSO) and D and $Q$ to eliminate senescent cells (magnification, 10X; $\mathrm{n}=3$ /group). RT-qPCR analysis of (E) $p 16^{\text {ink4a }}$ and $p 21^{\text {Cip1 }}$, and (F) Let-7f-5p, in BMSCs treated with vehicle (DMSO) and D and Q; gene expression was denoted as fold-change relative to vehicle ( $n=3$ /group). Data represent mean \pm SEM. ${ }^{*} p<0.05 ;{ }^{* *} p<0.01 ;{ }^{* * *} p<0.001$ (independent samples t-test).

Figure 5. Applicability of Let-7f as a control miRNA compared to U6. RT-qPCR analyses performed for miR-34a-5p using Let-7f and $U 6$ as endogenous control in (A) small needle bone biopsies obtained from the posterior iliac crest of 10 young (mean $\pm S D ; 27 \pm 3$ years) and 10 old (mean $\pm S D ; 78 \pm 6$ years) healthy female volunteers, (B) BMSCs treated with vehicle (DMSO) and Etoposide (20uM), and (C) BMSCs treated with vehicle and $D$ and $Q$; gene expression was denoted as fold-change relative to vehicle ( $n=3$ /group). Data represent mean \pm SEM. ${ }^{*} p<0.05$; ${ }^{* *} p<0.01$; ${ }^{* * *} p<0.001$ (independent samples t-test). 


\section{REFERENCES}

Allen-Rhoades, W., Kurenbekova, L., Satterfield, L., Parikh, N., Fuja, D., Shuck, R. L., Rainusso, N., Trucco, M., Barkauskas, D. A., Jo, E., Ahern, C., Hilsenbeck, S., Donehower, L. A., \& Yustein, J. T. (2015). Cross-species identification of a plasma microRNA signature for detection, therapeutic monitoring, and prognosis in osteosarcoma. Cancer Medicine, 4(7), 977-988.

https://doi.org/10.1002/cam4.438

Andersen, C. L., Jensen, J. L., \& Ørntoft, T. F. (2004). Normalization of real-time quantitative reverse transcription-PCR data: A model-based variance estimation approach to identify genes suited for normalization, applied to bladder and colon cancer data sets. Cancer Research, 64(15), 5245-5250. https://doi.org/10.1158/0008-5472.CAN-04-0496

Baker, D. J., Wijshake, T., Tchkonia, T., Lebrasseur, N. K., Childs, B. G., Sluis, B. Van De, Kirkland, J. L., \& Deursen, J. M. Van. (2011). Clearance of p16Ink4a-positive senescent cells delays ageingassociated disorders. Nature, 479(7372), 232-236. https://doi.org/10.1038/nature10600

Bartel, D. P. (2004). MicroRNAs: Genomics, Biogenesis, Mechanism, and Function. Cell, 116, $281-297$. https://doi.org/10.1016/j.bios.2017.04.001

Beyer, C., Zampetaki, A., Lin, N. Y., Kleyer, A., Perricone, C., lagnocco, A., Distler, A., Langley, S. R., Gelse, K., Sesselmann, S., Lorenzini, R., Niemeier, A., Swoboda, B., Distler, J. H. W., Santer, P., Egger, G., Willeit, J., Mayr, M., Schett, G., \& Kiechl, S. (2015). Signature of circulating microRNAs in osteoarthritis. Annals of the Rheumatic Diseases, 74(3), 1-7. https://doi.org/10.1136/annrheumdis2013-204698

Bignotti, E., Calza, S., Tassi, R. A., Zanotti, L., Bandiera, E., Sartori, E., Odicino, F. E., Ravaggi, A., Todeschini, P., \& Romani, C. (2016). Identification of stably expressed reference small non-coding RNAs for microRNA quantification in high-grade serous ovarian carcinoma tissues. Journal of Cellular and Molecular Medicine, 20(12), 2341-2348. https://doi.org/10.1111/jcmm.12927

Bottani, M., Banfi, G., \& Lombardi, G. (2019). Perspectives on mirnas as epigenetic markers in osteoporosis and bone fracture risk: A step forward in personalized diagnosis. Frontiers in Genetics, 10(OCT). https://doi.org/10.3389/fgene.2019.01044

Bushati, N., \& Cohen, S. M. (2007). MicroRNA functions. Annual Review of Cell and Developmental Biology, 23, 175-205. https://doi.org/10.1146/annurev.cellbio.23.090506.123406

Chen, J., Li, K., Pang, Q., Yang, C., Zhang, H., Wu, F., Cao, H., Liu, H., Wan, Y., Xia, W., Wang, J., Dai, Z., \& Li, Y. (2016). Identification of suitable reference gene and biomarkers of serum miRNAs for osteoporosis. Scientific Reports, 6(October), 1-11. https://doi.org/10.1038/srep36347 
Condrat, C. E., Thompson, D. C., Barbu, M. G., Bugnar, O. L., Boboc, A., Cretoiu, D., Suciu, N., Cretoiu, S. M., \& Voinea, S. C. (2020). miRNAs as Biomarkers in Disease: Latest Findings Regarding Their Role in Diagnosis and Prognosis. Cells, 1-32.

Costé, \& Rouleux-Bonnin, F. (2020). The crucial choice of reference genes: identification of miR-191-5p for normalization of miRNAs expression in bone marrow mesenchymal stromal cell and HS27a/HS5 cell lines. Scientific Reports, 10(1), 1-13. https://doi.org/10.1038/s41598-020-74685-7

Donati, S., Ciuffi, S., \& Brandi, M. L. (2019). Human circulating miRNAs real-time qRT-PCR-based analysis: An overview of endogenous reference genes used for data normalization. International Journal of Molecular Sciences, 20(18), 1-19. https://doi.org/10.3390/ijms20184353

Drobna, M., Szarzyńska-Zawadzka, B., Daca-Roszak, P., Kosmalska, M., Jaksik, R., Witt, M., \& Dawidowska, M. (2018). Identification of endogenous control mirnas for RT-qPCR in T-cell acute lymphoblastic leukemia. International Journal of Molecular Sciences, 19(10). https://doi.org/10.3390/ijms19102858

Farr, J. N., Fraser, D. G., Wang, H., Jaehn, K., Ogrodnik, M. B., Weivoda, M. M., Drake, M. T., Tchkonia, T., LeBrasseur, N. K., Kirkland, J. L., Bonewald, L. F., Pignolo, R. J., Monroe, D. G., \& Khosla, S. (2016). Identification of Senescent Cells in the Bone Microenvironment. Journal of Bone and Mineral Research, 31(11), 1920-1929. https://doi.org/10.1002/jbmr.2892

Farr, J. N., Roforth, M. M., Fujita, K., Nicks, K. M., Cunningham, J. M., Atkinson, E. J., Therneau, T. M., McCready, L. K., Peterson, J. M., Drake, M. T., Monroe, D. G., \& Khosla, S. (2015). Effects of age and estrogen on skeletal gene expression in humans as assessed by RNA sequencing. PLOS ONE, 10(9), 1-22. https://doi.org/10.1371/journal.pone.0138347

Farr, J. N., Xu, M., Weivoda, M. M., Monroe, D. G., Fraser, D. G., Onken, J. L., Negley, B. A., Sfeir, J. G., Ogrodnik, M. B., Hachfeld, C. M., Lebrasseur, N. K., Drake, M. T., Pignolo, R. J., Pirtskhalava, T., Tchkonia, T., Oursler, M. J., Kirkland, J. L., \& Khosla, S. (2017). Targeting cellular senescence prevents age-related bone loss in mice. Nat Med., 23(9), 1072-1079. https://doi.org/10.1038/nm.4385.Targeting

Foessl, I., Kotzbeck, P., \& Obermayer-Pietsch, B. (2019). miRNAs as novel biomarkers for bone related diseases. Journal of Laboratory and Precision Medicine, 4, 2-2. https://doi.org/10.21037/jpm.2018.12.06

Franceschi, C., Garagnani, P., Morsiani, C., Conte, M., Santoro, A., Grignolio, A., Monti, D., Capri, M., \& Salvioli, S. (2018). The continuum of aging and age-related diseases: Common mechanisms but different rates. Frontiers in Medicine, 5(MAR). https://doi.org/10.3389/fmed.2018.00061 
Fujita, K., Roforth, M. M., Demaray, S., McGregor, U., Kirmani, S., McCready, L. K., Peterson, J. M., Drake, M. T., Monroe, D. G., \& Khosla, S. (2014). Effects of estrogen on bone mRNA levels of sclerostin and other genes relevant to bone metabolism in postmenopausal women. Journal of Clinical Endocrinology and Metabolism, 99(1), 81-88. https://doi.org/10.1210/jc.2013-3249

Fulzele, S., Mendhe, B., Khayrullin, A., Johnson, M., Kaiser, H., Liu, Y., Isales, C. M., \& Hamrick, M. W. (2019). Muscle-derived miR-34a increases with age in circulating extracellular vesicles and induces senescence of bone marrow stem cells. Aging, 11(6), 1791-1803. https://doi.org/10.18632/aging.101874

Grillari, J., Mäkitie, R. E., Kocijan, R., Haschka, J., Vázquez, D. C., Semmelrock, E., \& Hackl, M. (2021). Circulating miRNAs in bone health and disease. Bone, 145(November 2020). https://doi.org/10.1016/j.bone.2020.115787

He, X., Zhang, W., Liao, L., Fu, X., Yu, Q., \& Jin, Y. (2013). Identification and Characterization of MicroRNAs by High Through-Put Sequencing in Mesenchymal Stem Cells and Bone Tissue from Mice of Age-Related Osteoporosis. PLOS ONE, 8(8). https://doi.org/10.1371/journal.pone.0071895

I. Mödder, U., Oursler, M. J., Khosla, S., \& Monroe, D. G. (2011). Wnt10b Activates the Wnt, Notch and NFKB Pathways in U2OS Osteosarcoma Cells. J Cell Biochem., 112(5), 1392-1402. https://doi.org/10.1002/jcb.23048.Wnt10b

Januszewski, A. S., Cho, Y. H., Joglekar, M. V., Farr, R. J., Scott, E. S., Wong, W. K. M., Carroll, L. M., Loh, Y. W., Benitez-Aguirre, P. Z., Keech, A. C., O’Neal, D. N., Craig, M. E., Hardikar, A. A., Donaghue, K. C., \& Jenkins, A. J. (2021). Insulin micro-secretion in Type 1 diabetes and related microRNA profiles. Scientific Reports, 11(1), 1-11. https://doi.org/10.1038/s41598-021-90856-6

Jaul, E., \& Barron, J. (2017). Age-Related Diseases and Clinical and Public Health Implications for the 85 Years Old and Over Population. Frontiers in Public Health, 5(December), 1-7. https://doi.org/10.3389/fpubh.2017.00335

Kaur, J., \& Farr, J. N. (2020). Cellular senescence in age-related disorders. Translational Research, 226, 96-104. https://doi.org/10.1016/j.trsl.2020.06.007

Kaur, J., Ferguson, S. L., Freitas, E., Miller, R., Bemben, D., Knehans, A., \& Bemben, M. (2019). Association of vitamin d status with chronic disease risk factors and cognitive dysfunction in $50-70$ year old adults. Nutrients, 11(1), 1-12. https://doi.org/10.3390/nu11010141

Khosla, S., Farr, J. N., Tchkonia, T., \& Kirkland, J. L. (2020). The role of cellular senescence in ageing and endocrine disease. Nature Reviews Endocrinology, 16(5), 263-275. https://doi.org/10.1038/s41574-020-0335-y 
Kirkland, J. L., \& Tchkonia, T. (2017). Cellular Senescence: A Translational Perspective. EBioMedicine, 21, 21-28. https://doi.org/10.1016/j.ebiom.2017.04.013

Landgraf, P., Rusu, M., Sheridan, R., Sewer, A., lovino, N., Aravin, A., Pfeffer, S., Rice, A., Kamphorst, A. O., Landthaler, M., Lin, C., Socci, N. D., Hermida, L., Fulci, V., Chiaretti, S., Foà, R., Schliwka, J., Fuchs, U., Novosel, A., ... Tuschl, T. (2007). A Mammalian microRNA Expression Atlas Based on Small RNA Library Sequencing. Cell, 129(7), 1401-1414. https://doi.org/10.1016/j.cell.2007.04.040

Lee, R., Feinbaum, R., \& Ambros, V. (1993). The C. elegans Heterochronic Gene lin-4 Encodes Small RNAs with Antisense Complementarity to lin-14. Cell, 116(116), 843-854.

Li, H., Fan, J., Fan, L., Li, T., Yang, Y., Xu, H., Deng, L., Li, J., Li, T., Weng, X., Wang, S., \& Zhao, R. C. (2018). MiRNA-10b reciprocally stimulates osteogenesis and inhibits adipogenesis partly through the TGF- $\beta /$ Smad2 signaling pathway. Aging and Disease, 9(6), 1058-1073.

https://doi.org/10.14336/AD.2018.0214

Li, Q., Wang, C., Cai, L., Lu, J., Zhu, Z., Wang, C., Su, Z., \& Lu, X. (2019). miR-34a derived from mesenchymal stem cells stimulates senescence in glioma cells by inducing DNA damage. Molecular Medicine Reports, 19(3), 1849-1857. https://doi.org/10.3892/mmr.2018.9800

Li, Y., Xiang, G. M., Liu, L. L., Liu, C., Liu, F., Jiang, D. N., \& Pu, X. Y. (2015). Assessment of endogenous reference gene suitability for serum exosomal microRNA expression analysis in liver carcinoma resection studies. Molecular Medicine Reports, 12(3), 4683-4691. https://doi.org/10.3892/mmr.2015.3919

Livak, K. J., \& Schmittgen, T. D. (2001). Analysis of relative gene expression data using real-time quantitative PCR and the 2- $\triangle \triangle C T$ method. Methods, 25(4), 402-408. https://doi.org/10.1006/meth.2001.1262

López-Otín, C., Blasco, M. A., Partridge, L., Serrano, M., \& Kroemer, G. (2013). The Hallmarks of Aging Europe PMC Funders Group. Cell, 153(6), 1194-1217. https://doi.org/10.1016/j.cell.2013.05.039

Lou, G., Ma, N., Xu, Y., Jiang, L., Yang, J., Wang, C., Jiao, Y., \& Gao, X. (2015). Differential distribution of U6 (RNU6-1) expression in human carcinoma tissues demonstrates the requirement for caution in the internal control gene selection for microRNA quantification. International Journal of Molecular Medicine, 36(5), 1400-1408. https://doi.org/10.3892/ijmm.2015.2338

Mandourah, A. Y., Ranganath, L., Barraclough, R., Vinjamuri, S., Hof, R. V. T., Hamill, S., Czanner, G., Dera, A. A., Wang, D., \& Barraclough, D. L. (2018). Circulating microRNAs as potential diagnostic biomarkers for osteoporosis. Scientific Reports, 8(1), 1-10. https://doi.org/10.1038/s41598-01826525-y 
Margaritis, K., Margioula-siarkou, G., Giza, S., Kotanidou, E. P., Tsinopoulou, V. R., Christoforidis, A., \& Galli-tsinopoulou, A. (2021). Micro-rna implications in type-1 diabetes mellitus: A review of literature. International Journal of Molecular Sciences, 22(22), 1-15. https://doi.org/10.3390/ijms222212165

Masè, M., Grasso, M., Avogaro, L., D’Amato, E., Tessarolo, F., Graffigna, A., Denti, M. A., \& Ravelli, F. (2017). Selection of reference genes is critical for miRNA expression analysis in human cardiac tissue. A focus on atrial fibrillation. Scientific Reports, 7(January), 1-10.

https://doi.org/10.1038/srep41127

Materozzi, M., Merlotti, D., Gennari, L., \& Bianciardi, S. (2018). The Potential Role of miRNAs as New Biomarkers for Osteoporosis. International Journal of Endocrinology, 2018. https://doi.org/10.1155/2018/2342860

McHugh, D., \& Gil, J. (2018). Senescence and aging: Causes, consequences, and therapeutic avenues. Journal of Cell Biology, 217(1), 65-77. https://doi.org/10.1083/jcb.201708092

Niu, Y., Wu, Y., Huang, J., Li, Q., Kang, K., Qu, J., Li, F., \& Gou, D. (2016). Identification of reference genes for circulating microRNA analysis in colorectal cancer. Scientific Reports, 6, 1-9. https://doi.org/10.1038/srep35611

Pfaffl, M. W., Tichopad, A., Prgomet, C., \& Neuvians, T. P. (2004). Determination of stable housekeeping genes, differentially regulated target genes and sample integrity: BestKeeper - Excel-based tool using pair-wise correlations. Biotechnology Letters, 26(6), 509-515. https://doi.org/10.1023/B:BILE.0000019559.84305.47

Pignolo, R. J., Law, S. F., \& Chandra, A. (2021). Bone Aging, Cellular Senescence, and Osteoporosis. JBMR Plus, 5(4), 1-14. https://doi.org/10.1002/jbm4.10488

Potter, M. L., Hill, W. D., Isales, C. M., Hamrick, M. W., \& Fulzele, S. (2021). MicroRNAs are critical regulators of senescence and aging in mesenchymal stem cells. Bone, 142(March 2020), 115679. https://doi.org/10.1016/j.bone.2020.115679

Ragni, E., Colombini, A., De Luca, P., Libonati, F., Viganò, M., Perucca Orfei, C., Zagra, L., \& de Girolamo, L. (2021). miR-103a-3p and miR-22-5p Are Reliable Reference Genes in Extracellular Vesicles From Cartilage, Adipose Tissue, and Bone Marrow Cells. Frontiers in Bioengineering and Biotechnology, 9(February), 1-15. https://doi.org/10.3389/fbioe.2021.632440

Rajeevan, M. S., Ranamukhaarachchi, D. G., Vernon, S. D., \& Unger, E. R. (2001). Use of real-time quantitative pcr to validate the results of CDNA array and differential display PCR technologies. Methods, 25(4), 443-451. https://doi.org/10.1006/meth.2001.1266

Schlosser, K., Mclntyre, L. A., White, R. J., \& Stewart, D. J. (2015). Customized internal reference 
controls for improved assessment of circulating micrornas in disease. PLOS ONE, 10(5), 1-22. https://doi.org/10.1371/journal.pone.0127443

Silver, N., Best, S., Jiang, J., \& Thein, S. L. (2006). Selection of housekeeping genes for gene expression studies in human reticulocytes using real-time PCR. BMC Molecular Biology, 7, 1-9. https://doi.org/10.1186/1471-2199-7-33

Song, J., Bai, Z., Han, W., Zhang, J., Meng, H., Bi, J., Ma, X., Han, S., \& Zhang, Z. (2012). Identification of suitable reference genes for QPCR analysis of serum microRNA in gastric cancer patients. Digestive Diseases and Sciences, 57(4), 897-904. https://doi.org/10.1007/s10620-011-1981-7

Thalyana, S. V., \& Slack, F. J. (2012). MicroRNAs and their roles in aging. Journal of Cell Science, 125(1), 7-17. https://doi.org/10.1242/jcs.099200

Vandesompele, J., Preter, K. De, Pattyn, F., Poppe, B., Roy, N. Van, Paepe, A. De, \& Speleman, F. (2002). Accurate normalization of real-time quantitative RT-PCR data by geometric averaging of multiple internal control genes. Genome Biology, 3(7), research0034.1-0034.11 The. https://doi.org/10.1007/s00603-018-1496-z

Wang, L., Liu, Y., Du, L., Li, J., Jiang, X., Zheng, G., Qu, A., Wang, H., Wang, L., Zhang, X., Liu, H., Pan, H., Yang, Y., \& Wang, C. (2015). Identification and validation of reference genes for the detection of serum microRNAs by reverse transcription-quantitative polymerase chain reaction in patients with bladder cancer. Molecular Medicine Reports, 12(1), 615-622. https://doi.org/10.3892/mmr.2015.3428

Wightman, B., Ha, I., \& Ruvkun, G. (1993). Posttranscriptional regulation of the heterochronic gene lin-14 by lin-4 mediates temporal pattern formation in C. elegans. Cell, 75(5), 855-862. https://doi.org/10.1016/0092-8674(93)90530-4

Xiang, M., Zeng, Y., Yang, R., Xu, H., Chen, Z., Zhong, J., Xie, H., Xu, Y., \& Zeng, X. (2014). U6 is not a suitable endogenous control for the quantification of circulating microRNAs. Biochemical and Biophysical Research Communications, 454(1), 210-214. https://doi.org/10.1016/j.bbrc.2014.10.064

Xu, M., Tchkonia, T., Ding, H., Ogrodnik, M., Lubbers, E. R., Pirtskhalava, T., White, T. A., Johnson, K. O., Stout, M. B., Mezera, V., Giorgadze, N., Jensen, M. D., LeBrasseur, N. K., \& Kirkland, J. L. (2015). JAK inhibition alleviates the cellular senescence-associated secretory phenotype and frailty in old age. Proceedings of the National Academy of Sciences of the United States of America, 112(46), E6301-E6310. https://doi.org/10.1073/pnas.1515386112

Xu, T., Luo, Y., Wang, J., Zhang, N., Gu, C., Li, L., Qian, D., Cai, W., Fan, J., \& Yin, G. (2020). Exosomal miRNA-128-3p from mesenchymal stem cells of aged rats regulates osteogenesis and bone fracture 
healing by targeting Smad5. Journal of Nanobiotechnology, 18(1), 1-18.

https://doi.org/10.1186/s12951-020-00601-w

Zhang, W., Hsu, P., Zhong, B., Guo, S., Zhang, C., Wang, Y., Luo, C., Zhan, Y., \& Zhang, C. (2018). MiR34a Enhances Chondrocyte Apoptosis, Senescence and Facilitates Development of Osteoarthritis by Targeting DLL1 and Regulating PI3K/AKT Pathway. Cellular Physiology and Biochemistry, 48(3), 1304-1316. https://doi.org/10.1159/000492090

Zhou, Y., Xin, X., Wang, L., Wang, B., Chen, L., Liu, O., Rowe, D. W., \& Xu, M. (2021). Senolytics improve bone forming potential of bone marrow mesenchymal stem cells from aged mice. Npj Regenerative Medicine, 6(1), 1-5. https://doi.org/10.1038/s41536-021-00145-z

Zhu, Y., Tchkonia, T., Pirtskhalava, T., Gower, A. C., Ding, H., Giorgadze, N., Palmer, A. K., Ikeno, Y., Hubbard, G. B., Lenburg, M., O'hara, S. P., Larusso, N. F., Miller, J. D., Roos, C. M., Verzosa, G. C., Lebrasseur, N. K., Wren, J. D., Farr, J. N., Khosla, S., ... Kirkland, J. L. (2015). The achilles' heel of senescent cells: From transcriptome to senolytic drugs. Aging Cell, 14(4), 644-658. https://doi.org/10.1111/acel.12344 


\section{Figure 1}

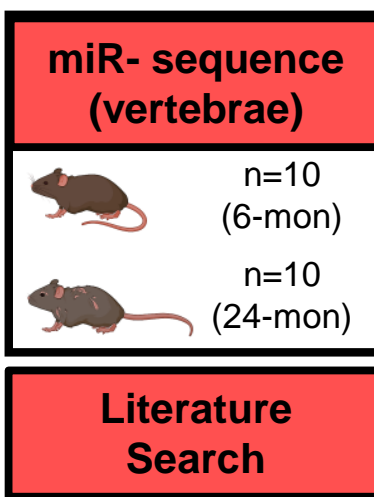

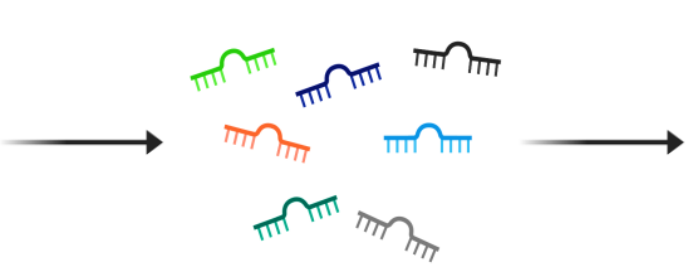

Candidate Endogenous Control miRNAs

\section{GeNorm}

NormFinder

BestKeeper

Comparative Delta CT

RefFinder

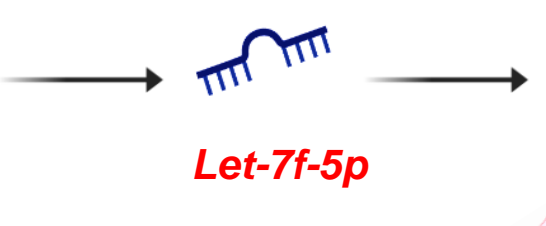

i

Senolytics

Let-7f-5p versus U6 


\section{Figure 2}

A

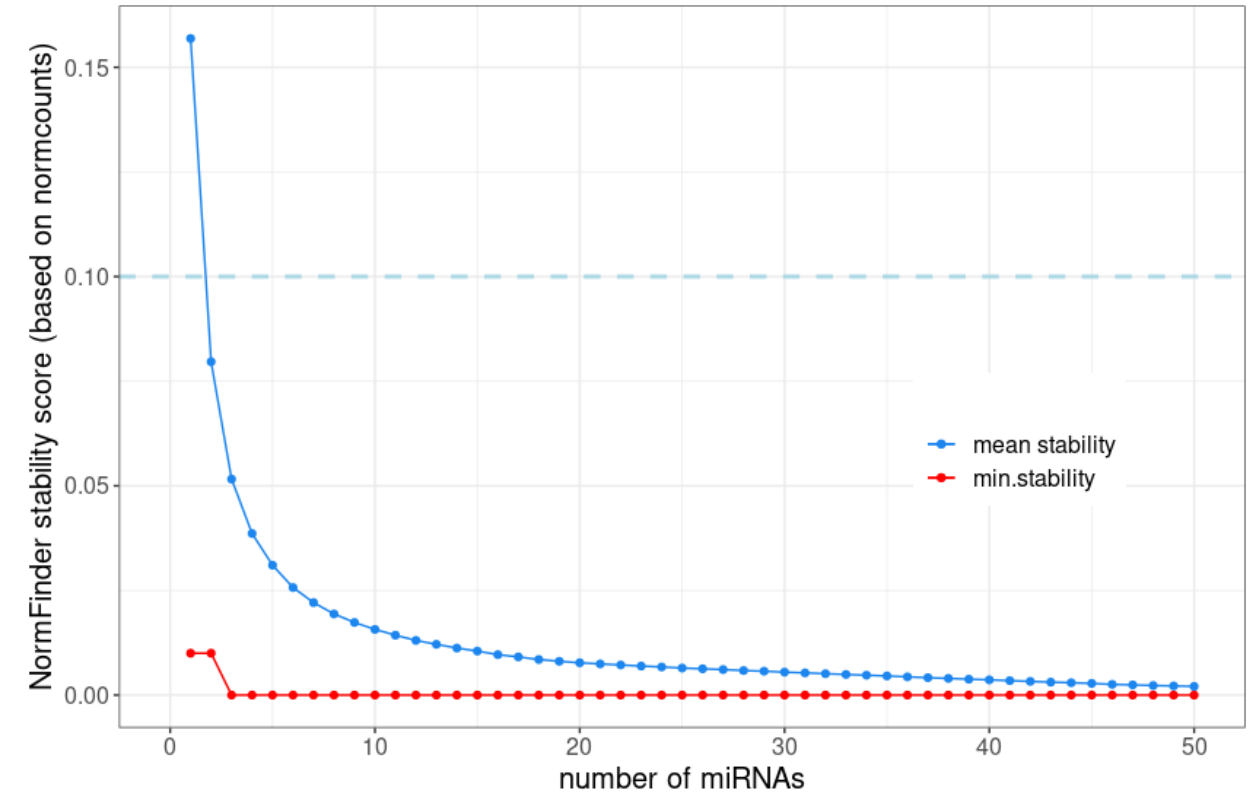

B

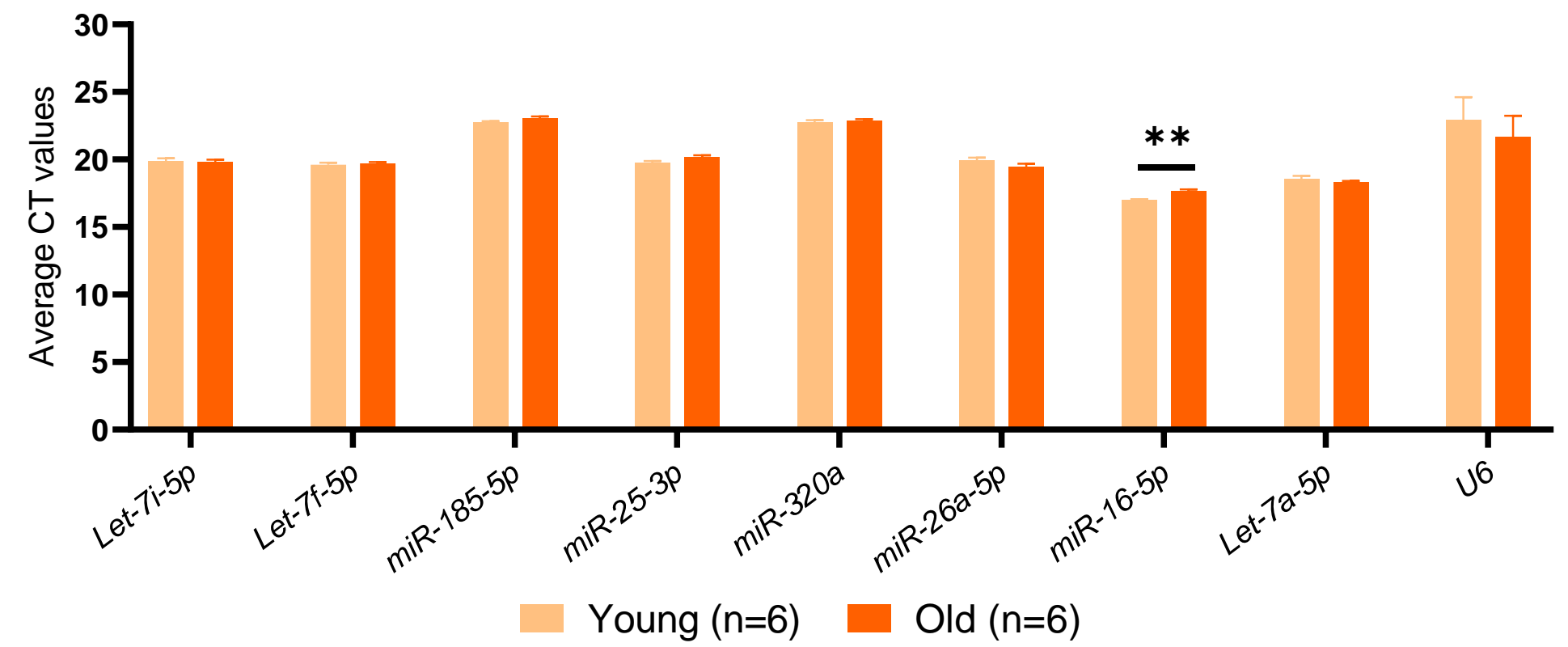




\section{Figure 3}

\section{A \\ GeNorm \\ B NormFinder}

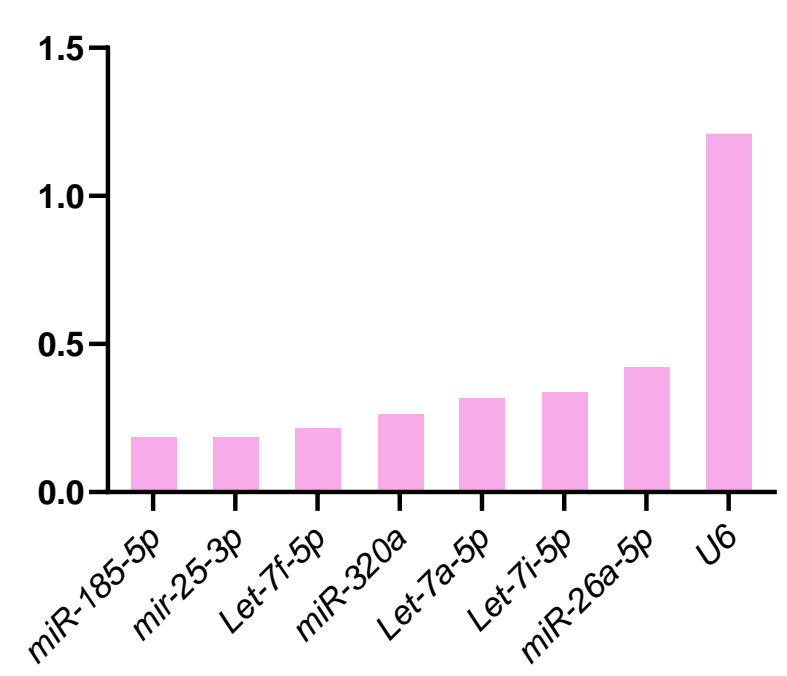

E

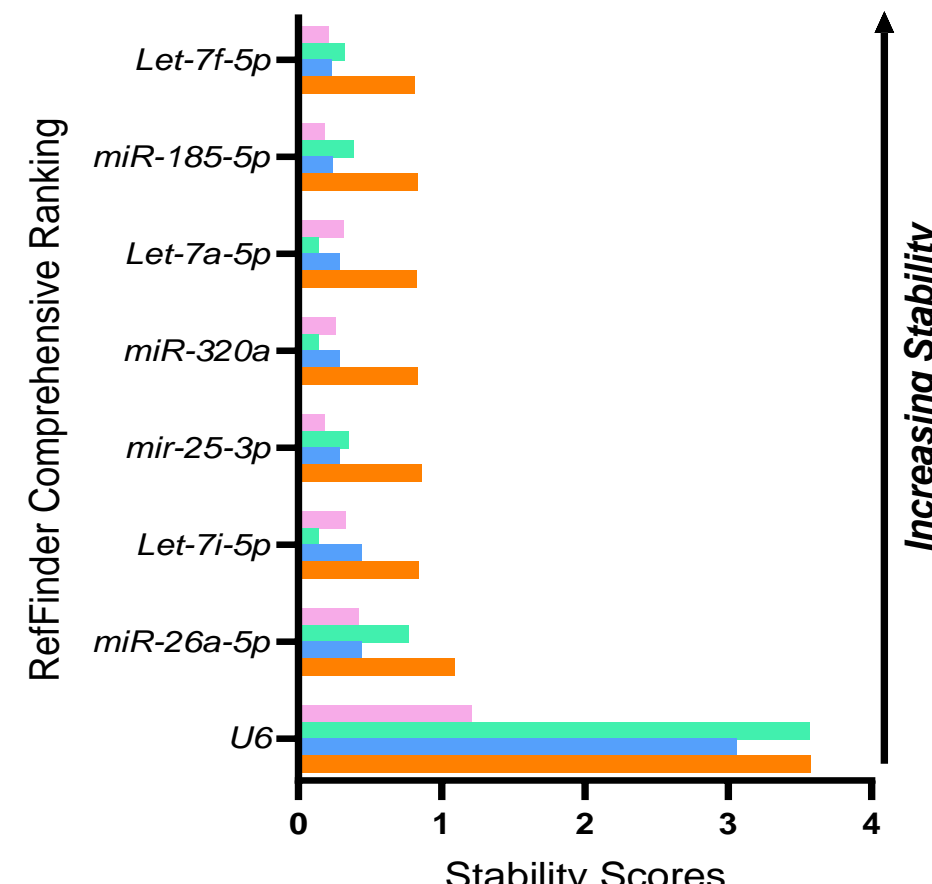

GeNorm

NormFinder

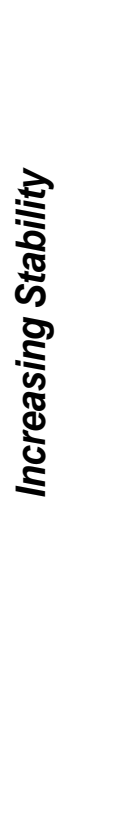

BestKeeper

deltaCT

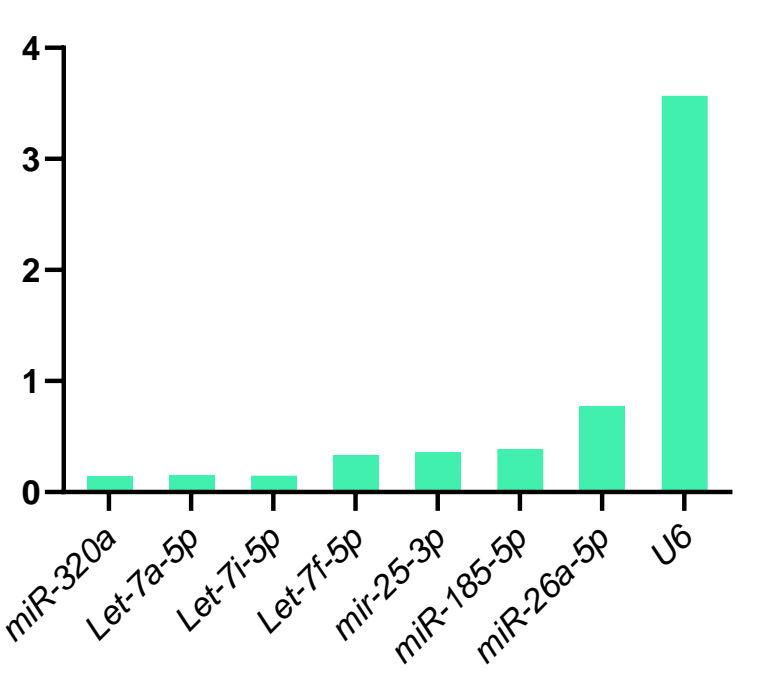

F

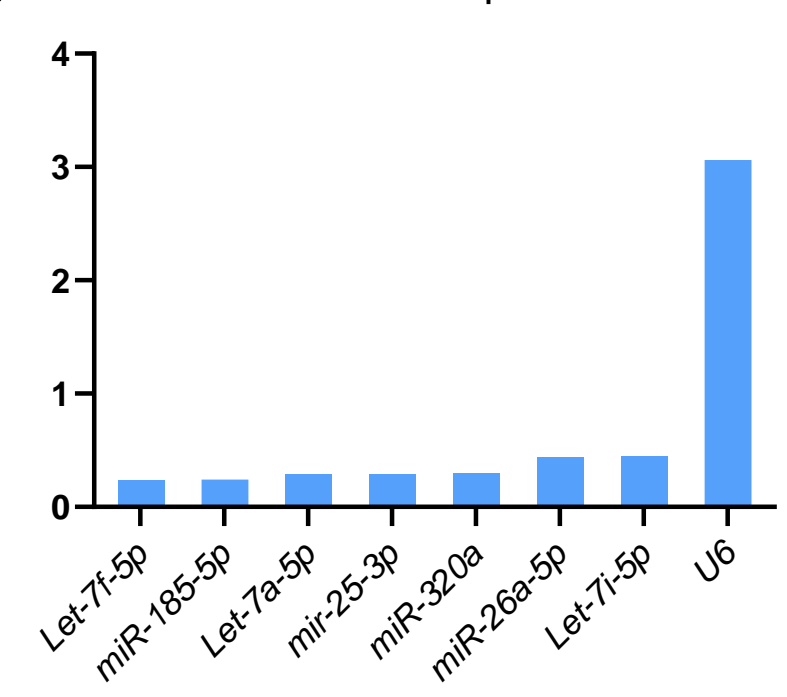

Let-7f-5p

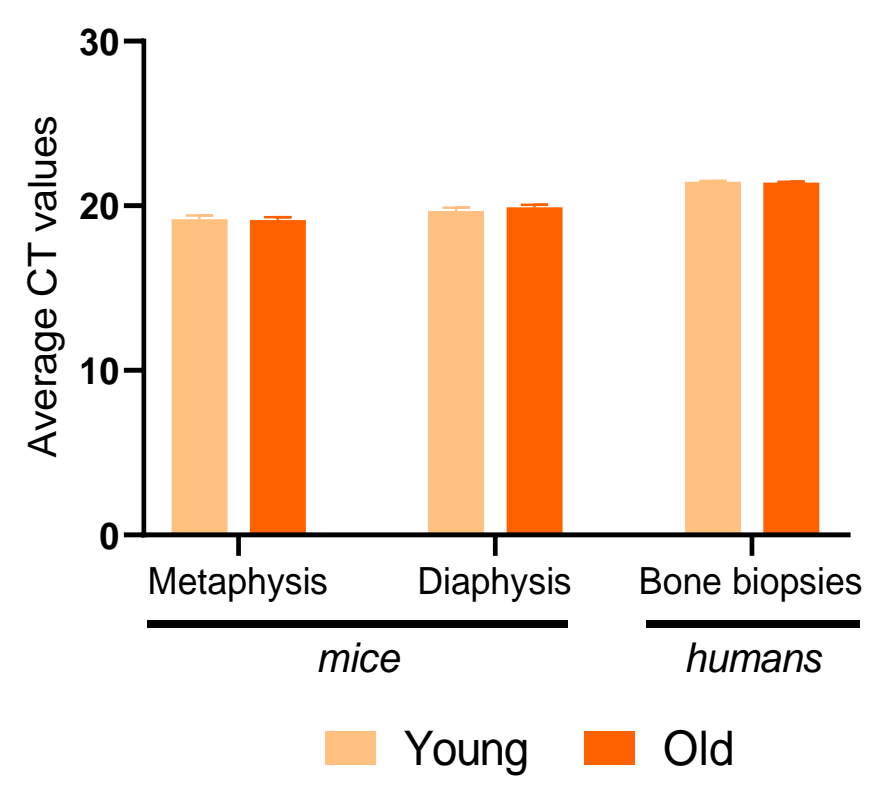

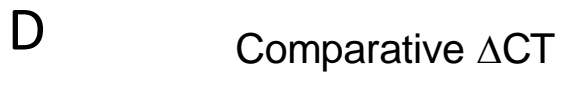

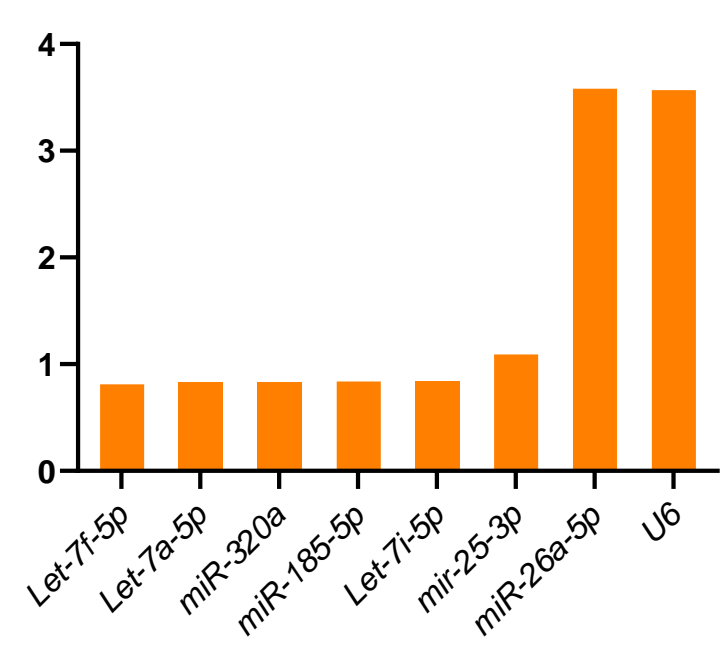


Figure 4

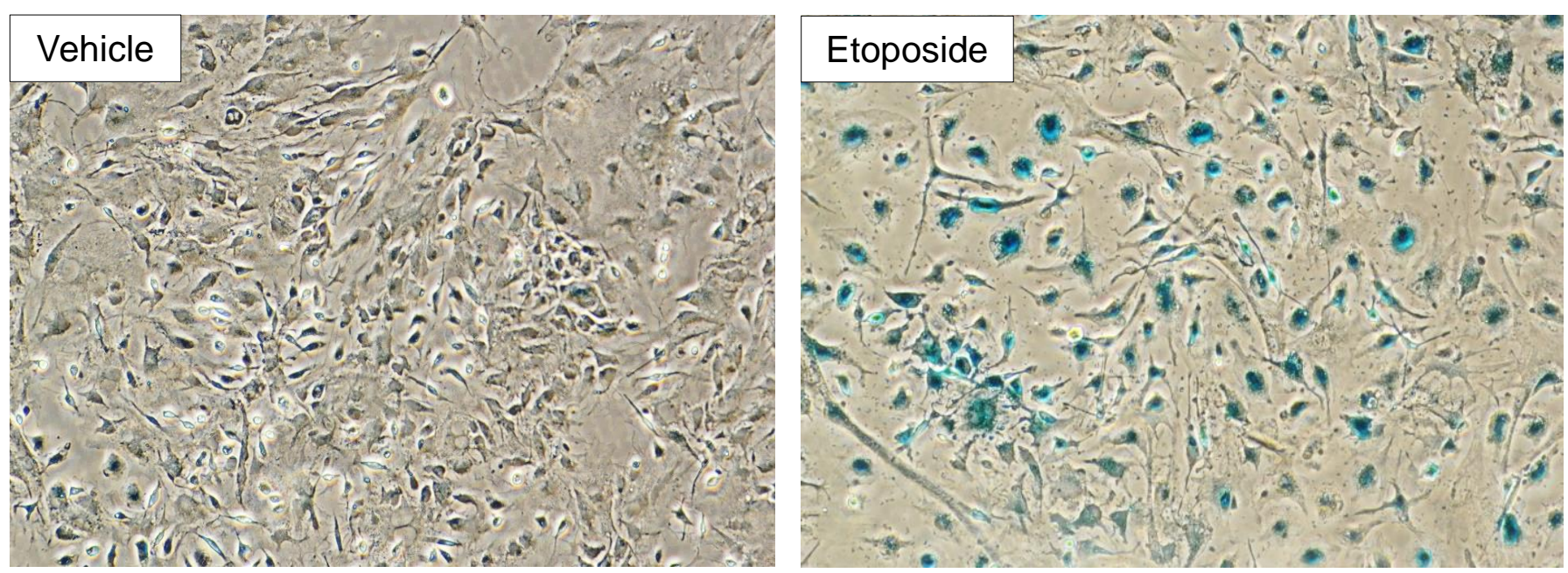

D
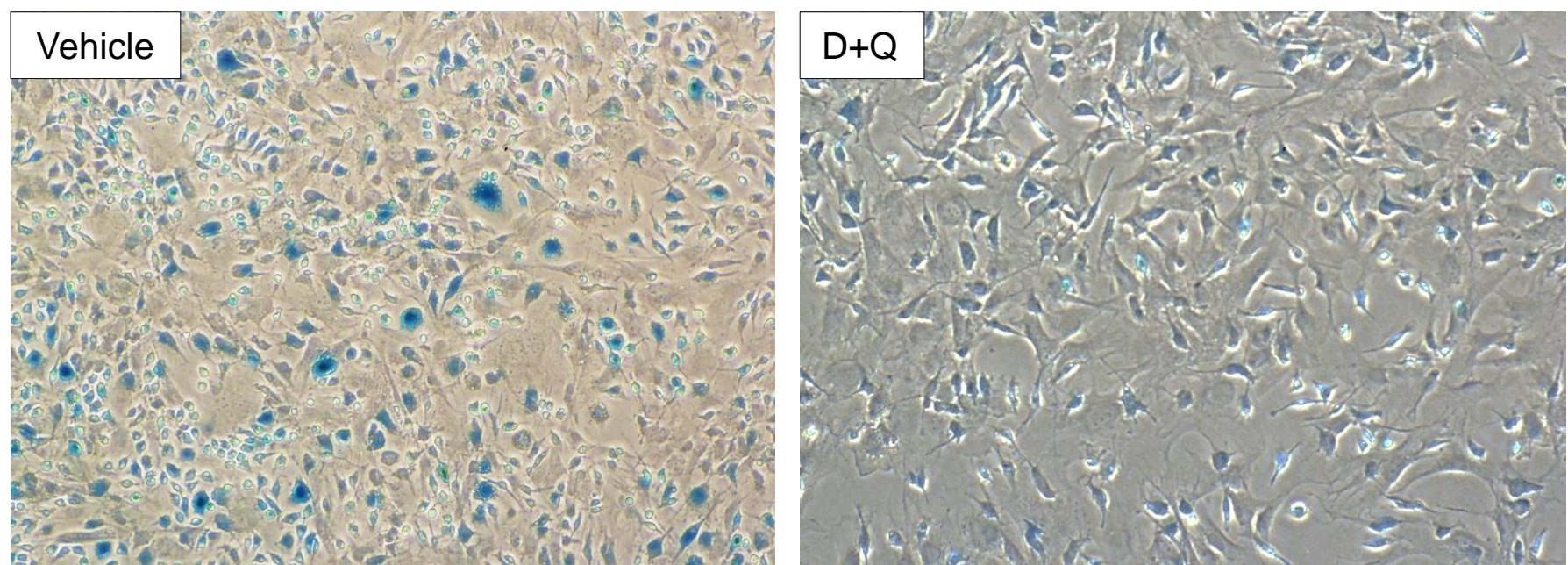

B

C
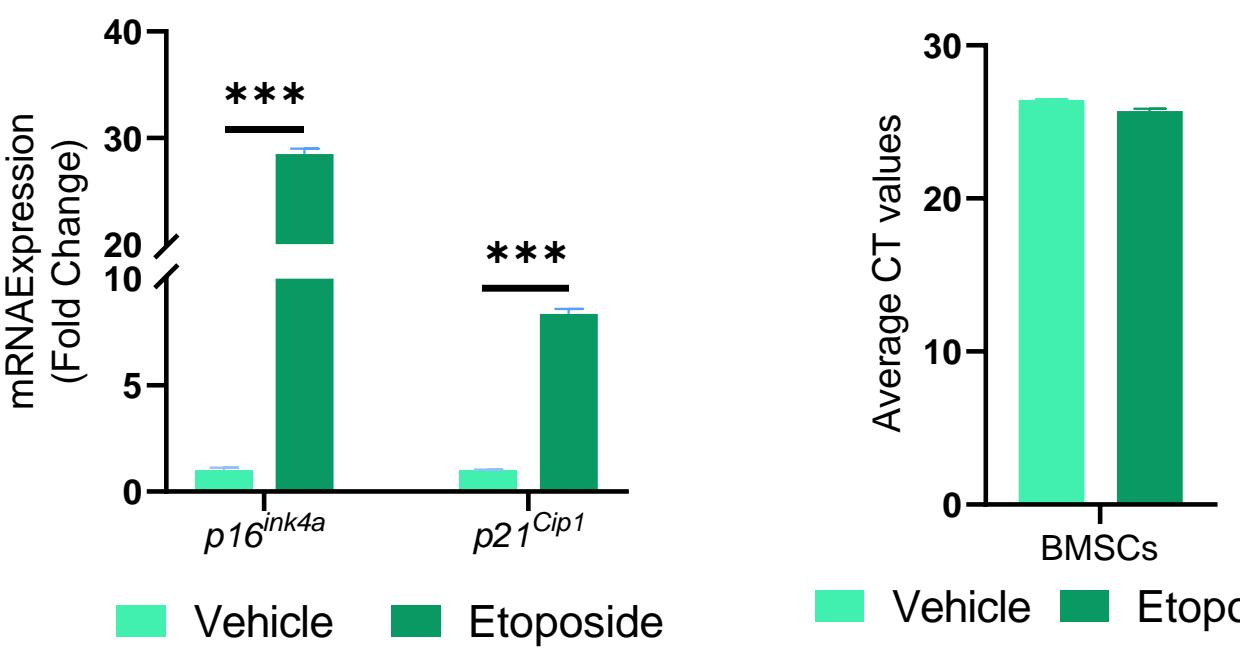

- Vehicle Etoposide

E

F

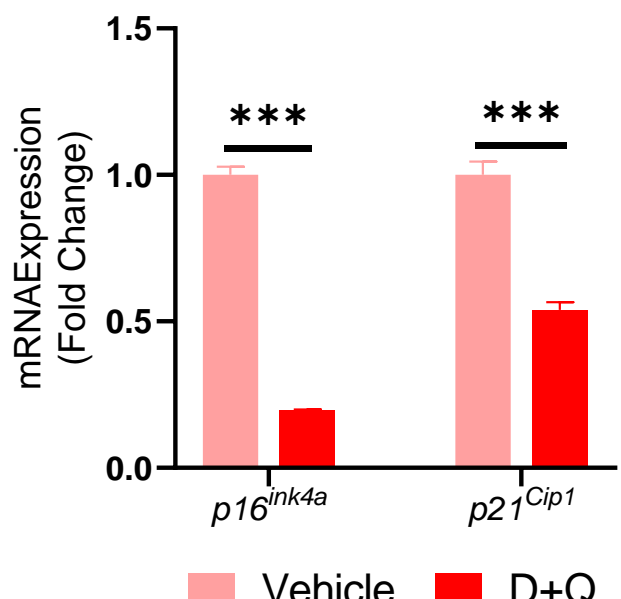

Let-7f-5p

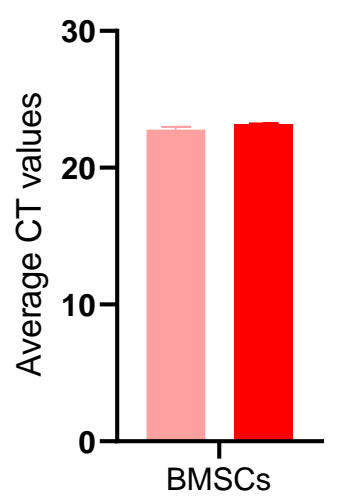

- Vehicle $\mathrm{D}+\mathrm{Q}$ 


\section{Figure 5}

A

miR-34a-5p

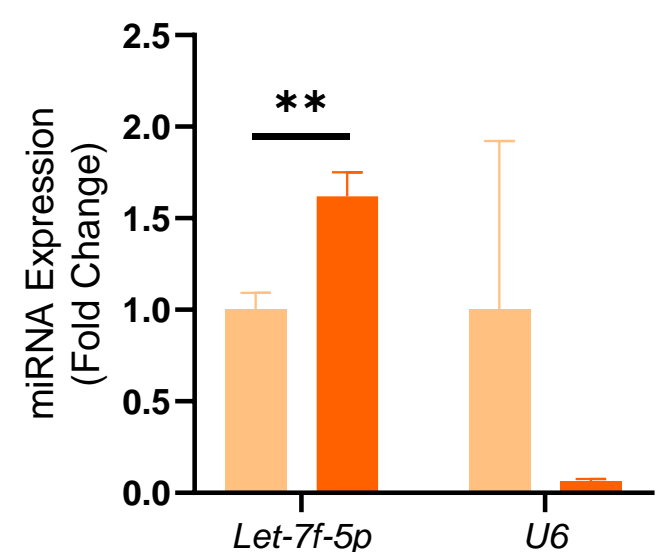

Young (humans) $\quad$ Old (humans)
B

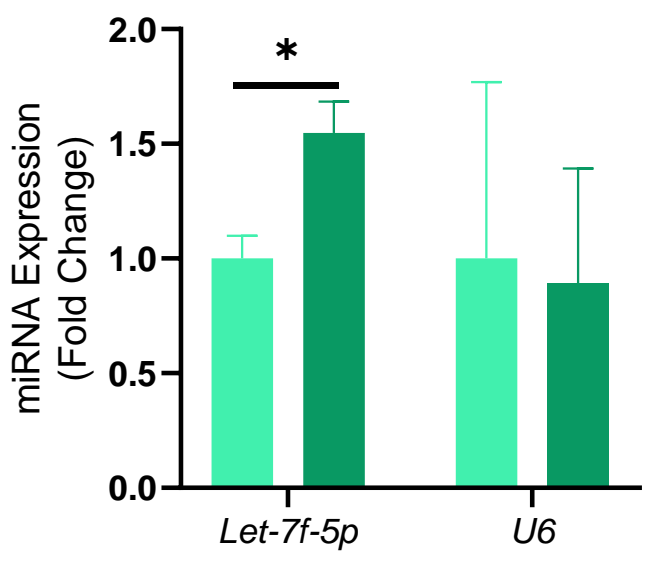

Vehicle Etoposide
C $\quad$ miR-34a-5p

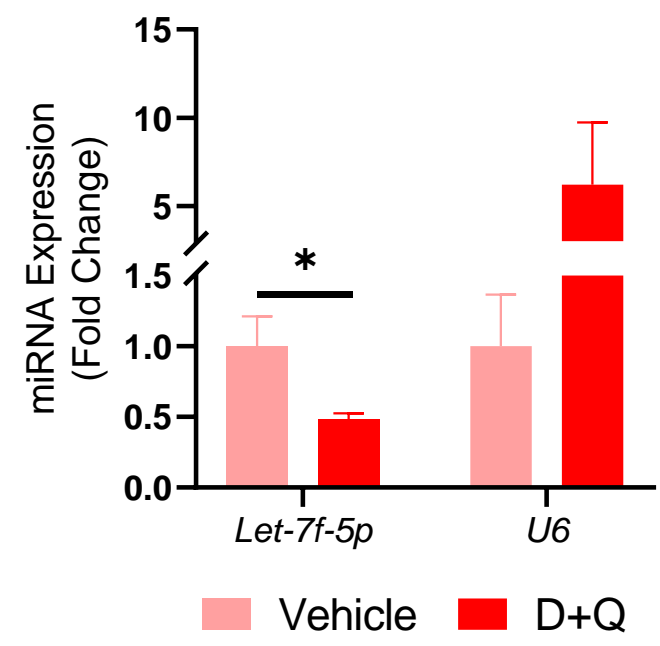


Table 1. Candidate endogenous control miRNAs.

\begin{tabular}{|c|c|c|c|}
\hline miRNA & Normalized Count & $\begin{array}{l}\text { NormFinder } \\
\text { Stability Score }\end{array}$ & References \\
\hline Let-7i-5p* & 87679.67 & 0.07 & - \\
\hline Let-7f-5p* & 47232.57 & 0.16 & - \\
\hline$m i R-185-5 p^{*}$ & 971.41 & 0.09 & - \\
\hline$m i R-25-3 p \pi$ & 10084.71 & 0.21 & Chen et al., 2016; Drobna et al., 2018; Niu et al., 2016 \\
\hline miR-320al \# & - & - & Allen-Rhoades et al., 2015; Schlosser et al., 2015 \\
\hline$m i R-26 a-5 p^{\prime}$ & 21518.389 & 0.11 & Y. Li et al., 2015 \\
\hline$m i R-16-5 p^{\pi}$ & 1712.7345 & 0.27 & Drobna et al., 2018; Song et al., 2012; Wang et al., 2015 \\
\hline Let-7a-5p & 8940.0595 & 0.27 & Drobna et al., 2018 \\
\hline
\end{tabular}

*Selected from miRNA sequence data based on normalized counts and stability scores. "Selected from literature search. The normalized counts and stability scores based on miRNA sequence data are mentioned for reference. " miRNA not expressed in miRNA sequence data. 